\title{
An integrated pathway interaction network for the combination of four effective compounds from ShengMai preparations in the treatment of cardio- cerebral ischemic diseases
}

\author{
Fang LI\#, Yan-ni LV\#, Yi-sha TAN, Kai SHEN, Ke-feng ZHAl, Hong-lin CHEN, Jun-ping KOU*, Bo-yang YU* \\ Jiangsu Key Laboratory of TCM Evaluation and Translational Research, Department of Complex Prescription of TCM, China \\ Pharmaceutical University, Nanjing 211198, China
}

\begin{abstract}
Aim: SMXZF (a combination of ginsenoside Rb1, ginsenoside Rg1, schizandrin and DT-13) derived from Chinese traditional medicine formula ShengMai preparations) is capable of alleviating cerebral ischemia-reperfusion injury in mice. In this study we used network pharmacology approach to explore the mechanisms of SMXZF in the treatment of cardio-cerebral ischemic diseases.

Methods: Based upon the chemical predictors, such as chemical structure, pharmacological information and systems biology functional data analysis, a target-pathway interaction network was constructed to identify potential pathways and targets of SMXZF in the treatment of cardio-cerebral ischemia. Furthermore, the most related pathways were verified in TNF- $\alpha$-treated human vascular endothelial EA.hy926 cells and $\mathrm{H}_{2} \mathrm{O}_{2}$-treated rat PC12 cells.

Results: Three signaling pathways including the NF-kB pathway, oxidative stress pathway and cytokine network pathway were demonstrated to be the main signaling pathways. The results from the gene ontology analysis were in accordance with these signaling pathways. The target proteins were found to be associated with other diseases such as vision, renal and metabolic diseases, although they exerted therapeutic actions on cardio-cerebral ischemic diseases. Furthermore, SMXZF not only dose-dependently inhibited the phosphorylation of NF-KB, p50, p65 and IKK $\alpha / \beta$ in TNF- $\alpha$-treated EA.hy926 cells, but also regulated the $\mathrm{Nrf}_{2} / \mathrm{HO}^{-1}$ pathway in $\mathrm{H}_{2} \mathrm{O}_{2}{ }^{-}$ treated PC12 cells.

Conclusion: NF-kB signaling pathway, oxidative stress pathway and cytokine network pathway are mainly responsible for the therapeutic actions of SMXZF against cardio-cerebral ischemic diseases.
\end{abstract}

Keywords: ShengMai San; cardio-cerebral ischemic diseases; network pharmacology; traditional Chinese medicines; target-pathway interaction network; gene ontology; NF-kB; oxidative stress; EA.hy926 cells; PC12 cells

Acta Pharmacologica Sinica (2015) 36: 1337-1348; doi: 10.1038/aps.2015.70; published online 12 Oct 2015

\section{Introduction}

Traditional Chinese medicine (TCM) has been used in clinical practice for thousands of years and has accumulated considerable knowledge concerning the in vivo efficacy of targeting complicated diseases. TCM formulae are a mixture of hundreds of chemical components with multiple potential targets, essentially acting as a combination therapy of multicomponent drugs ${ }^{[1]}$. However, the unclear molecular mechanisms of TCMs are obstacles to their further development and

\footnotetext{
\# These two authors contributed equally to this work.

* To whom correspondence should be addressed.

E-mail junpingkou@cpu.edu.cn (Jun-ping KOU); boyangyu59@163.com (Bo-yang YU)

Received 2015-04-19 Accepted 2015-07-12
}

internationalization. The fast development of network-based systems biology has facilitated an understanding of the interactions among genes and proteins at a holistic level and represents new methods for uncovering the molecular mechanisms related to the therapeutic efficacy of $\mathrm{TCM}^{[2-4]}$.

Typically, the drug-target association network has been applied to elucidate the action mechanism of drugs ${ }^{[5]}$. Meanwhile, the drug-drug network and the protein-disease network have also been employed to discover the association between diseases and target proteins ${ }^{[6]}$. Theoretical algorithms such as CIPHER have been proposed to identify pathways and indicate network-based computational frameworks ${ }^{[7]}$. Using these methods, the proteins used in analysis were usually chosen from the classic disease-related proteins or based on the docking scores or term frequencies of compounds ${ }^{[5]}$. The experi- 
mental verification typically focused on the signaling pathways, which served as the best biomarker compared to single proteins. However, key proteins identified by the common methods introduced difficulties in experimental verification because multiple key proteins might not be related, as they may reside in different signaling pathways. Therefore, we proposed that the interpretation of the target-pathway interaction network mechanism might be an alternative approach for target discovery. The target-pathway interaction network (Figure 1) clusters the signaling pathways based on the highdegree nodes in the drug-target network. Then, the potential important proteins are identified from the highly scored clustered pathways, and these proteins have a high degree of robustness within the network and contribute significantly to the highly clustered pathway. Thus, the identification of key proteins via the target-pathway interaction network not only facilitates the verification of limited main interacting signaling pathways but also increases the validity of key targets.

ShengMai San is a traditional Chinese formula and its remedy preparations have already been directly used to prevent and cure cardio-cerebral ischemic diseases ${ }^{[8-10]}$. Several main types of chemical components, such as ginsenosides, lignans and homoisoflavonoids, have been suggested to be responsible for the pharmacological activities of ShengMai preparations ${ }^{[11]}$. In our previous work, we screened a new combination (SMXZF) comprising four compounds, including ginsenoside $\mathrm{Rb} 1$, ginsenoside Rg1, DT-13 and schisandrin (the proportion as 6: 9:4:5), that showed a significant inhibition of cardio-cerebral ischemia in experimental studies ${ }^{[12,13]}$. Ginsenoside Rb1 and Rg1 are ginsenosides, which have been widely reported to protect against cardiovascular diseases ${ }^{[14]}$. The schisandrin from the lignans exhibits a neuroprotective effect on glutamate-induced neuronal excitotoxicity ${ }^{[15]}$. DT-13 is an ophio- pogonins and has been reported to reduce calcium currents during hypoxia in adult rat ventricular myocytes ${ }^{[16]}$. Therefore, SMXZF is considered to be potentially useful because the safety and validity of its four components in cardio-cerebral ischemia have been reported. However, the action mode and related signaling pathways of SMXZF are unknown.

To obtain a better understanding of how the combination affects biological processes, the target-pathway interaction network was used to interpret the relationship of the targets and pathways of SMXZF. Then, the most related pathways were verified in EA.hy926 endothelial cells (ECs) stimulated by tumor necrosis factor- $\alpha$ (TNF- $\alpha$ ) and in PC12 cells activated by hydrogen peroxide $\left(\mathrm{H}_{2} \mathrm{O}_{2}\right)$. The endothelium plays a critical role in the regulation of leukocyte adhesion, platelet activity and thrombosis, which are all involved in the development of cardio-cerebral diseases ${ }^{[17]}$. Moreover, dysfunction of the endothelial cells induced by TNF- $a$, such as endothelial activation and vascular inflammation, plays an essential role in the pathogenesis of cardio-cerebral vascular diseases ${ }^{[18]}$. Rat pheochromocytoma PC12 cells have been widely used as a model for studies of neuronal function and stroke-related diseases ${ }^{[19]}$. Oxidative stress-induced cell damage mediated by $\mathrm{H}_{2} \mathrm{O}_{2}$ has been implicated in a variety of neurodegenerative disorders such as stroke and Alzheimer's disease ${ }^{[20,21]}$. Our findings will shed new light on the pharmacological mechanism of the combination derived from ShengMai preparations and will facilitate an understanding of the detailed mechanisms of cardiocerebral diseases in the network context.

\section{Materials and methods}

\section{Construction of the protein database}

To identify the corresponding gene targets of the active components from ShengMai preparations, we selected genes that
A

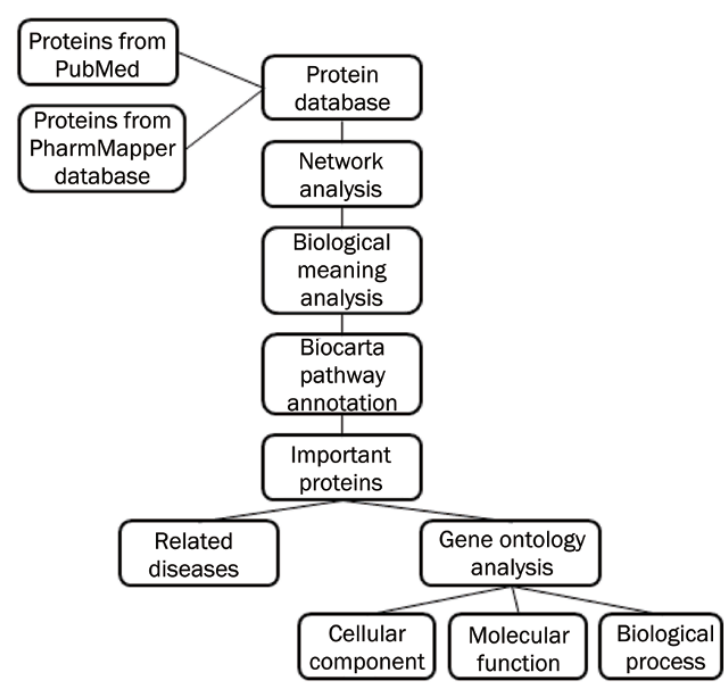

B

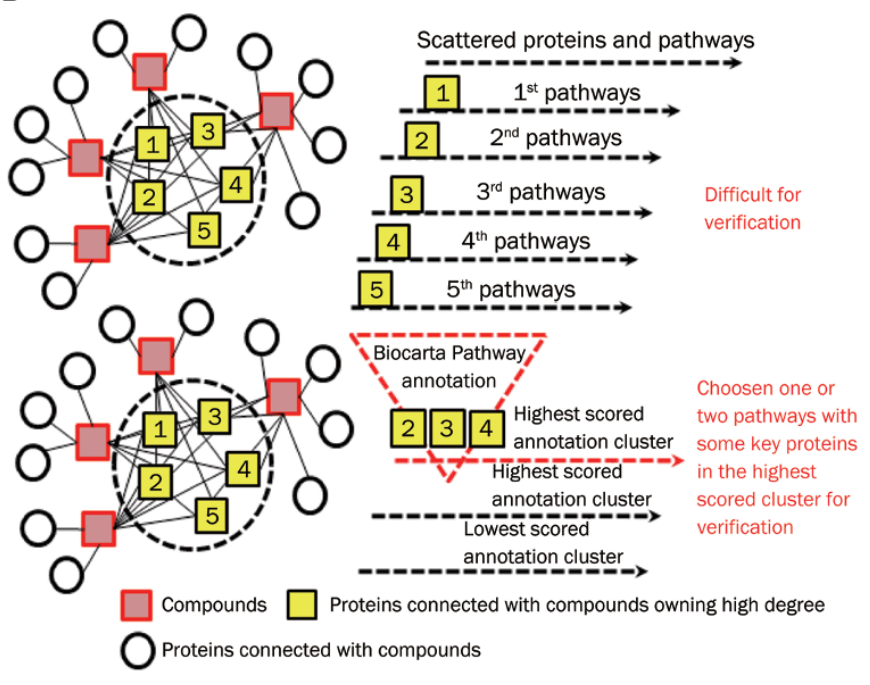

Figure 1. Network pharmacology analysis workflow. (A) The flow diagrams based on the target-pathway interaction work. (B) The comparison between the target-pathway interaction network and the traditional network. The 1st to 5th pathways represent different annotation clusters analyzed by different methods. 
had interactions with these compounds. The protein database was made up of two parts. The first part of the database on known targets was derived from the literatures from PubMed Central of the NCBI database (http:/ / www.ncbi.nlm.nih.gov/ pubmed/). The search terms comprised a compound name (ginsenoside Rb1 or ginsenoside Rg1 or DT-13 or schisandrin) and key words of diseases. The key words of diseases were standardized using Mesh terms in the NCBI database (http://www.ncbi.nlm.nih.gov/mesh/), including heart or myocardial ischemia, cerebral ischemia or infarction, or brain ischemia and stroke. As an example, the following search term was used "ginsenoside Rg1 and heart ischemia". The proteins/genes that conformed to the Mesh terms of diseases were text mined from the abstracts to construct the network. The proteins/genes/GEO profiles were standardized in the NCBI database, "Homo sapiens". The second part served as a supplement to the database, including high affinity and novel proteins related to the compounds. The targets were derived from the PharmMapper database (http://59.78.96.61/pharmmapper/), which was designed to predict potential target candidates for small molecules via a reverse pharmacophore mapping approach. PharmMapper is backed up by a large, in-house pharmacophore database covering over 7,000 receptor-based pharmacophore models, 459 of which are human protein targets ${ }^{[22]}$. Structures of the four compounds were constructed by Sybyl 6.9 and were applied to all hydrogens and Gastiger-Huckel charges in Sybyl 6.9 in the Tripos force field in an implicit solvent environment. The minimization steps included 1000 cycles of steepest descent until the convergent threshold of $0.05 \mathrm{kal}^{-1} \cdot \AA^{-1}$ was satisfied. Four minimized molecules were loaded into the PharmMapper human protein database, and the top ranking 100 proteins were selected as the matched hits using the default settings. One hundred proteins had standardized proteins/gene/GEO file names (http://www.ncbi.nlm.nih.gov/geoprofiles/) in Homo sapiens. The combined parts of the protein database were filtered in relation to cardio-cerebral ischemia in PubMed Central in the NCBI database with search terms including the protein/gene name and key words for the diseases. The proteins/genes that were related to the diseases were included in the second part of the database.

\section{The construction and analysis of the network}

We constructed the drug-target network using Cytoscape $2.8 .3^{[23,24]}$, which was developed for the reconstruction and visualization of networks. Cytoscape is a desktop Java application and is available for download from http://cytoscape. org. The drug-target network was constructed and we analyzed the degree and betweenness of the compounds in the network with the Centiscape module. The most elementary characteristic of a node was its degree, which told us how many links the node had to other nodes. The degree and the betweenness were the node topological and central indexes, and the potential biological meanings of higher-degree nodes were tested via Biocarta functional annotation analysis ${ }^{[25]}$. Higher degree and larger betweenness nodes are most impor- $\operatorname{tant}{ }^{[26,27]}$. Ginsenoside Rb1, ginsenoside Rg1, and schisandrin have been widely reported because of their functions in related diseases, and we chose nodes with connection degrees greater or equal to the average connection degree of the drugtarget network as part of an important protein group. Thus, a degree $\geq 3$ was preferred in this analysis of the main signaling pathways. Pathway analysis always plays an essential role in discovering possible biological processes that the genes from the input list participate in. Many databases such as Biocarta, KEGG and GeneGo collect cured pathways, including those of cellular processes, metabolism and diseases ${ }^{[28]}$. In this study, the signaling pathways of new sets were identified based on pathway interaction networks collected using Biocarta. The clustered pathway required enriched functions for genes when Fisher's exact $P$-value $\leq 0.01$. The functional annotation cluster was constructed of pathways representing similar pathways according to the enrichment scores. Gene ontology (GO), another type of pathway, typically reveals the physiological functions and cellular locations of a group of genes and is applied to identify enriched GO function for genes in the network ${ }^{[25]}$. GO annotations were performed using the BINGO 2.4.4 plugin of Cytoscape 2.8.3. A statistical test (a hypergeometric test), a multiple testing correction (Benjamini and Hochberg's FDR correction) and a significance level (0.05) were selected. The overrepresentation of functional categories and Homo sapiens annotation was assessed in our clusters. The genes were subjected to biological process, cellular component and molecular function ontology file analysis. The association between the important proteins and other diseases was also discussed according to the disease-associated network using the genetic association database.

\section{Cell culture and drug treatments}

The endothelial cell line EA.hy926 and rat pheochromocytoma PC12 cells were purchased from the Cell Bank of the Chinese Academy of Sciences (Shanghai, China). The cells were grown in Dulbecco's modified Eagle's medium (DMEM) (Life Technologies, CA, USA) supplemented with $10 \%$ fetal bovine serum (Wisent, Nanjing, China), $100 \mathrm{U} / \mathrm{mL}$ penicillin and 100 $\mu \mathrm{g} / \mathrm{mL}$ streptomycin at $37^{\circ} \mathrm{C}$ in a humidified atmosphere containing $5 \% \mathrm{CO}_{2}$. The cells were seeded onto 6-well plates for the administration of drug treatments when grown to anastomose.

SMXZF was a mixture of ginsenoside Rb1, Rg1, schisandrin and DT-13 (6:9:5:4). Ginsenoside Rb1, Rg1 and schisandrin were purchased from Nanjing Zelang Bio-Technology Co, Ltd (Nanjing, China). DT-13 was isolated in our own laboratory. The purity of each compound was determined to be higher than $98 \%$ by HPLC. SMXZF and N-acetyl cysteine (NAC) (Sigma-Aldrich, St Louis, MO, USA) were dissolved in dimethyl sulfoxide and then diluted in the serum free medium. Then, the endothelial cells were treated with 0.1 $10 \mu \mathrm{g} / \mathrm{mL}$ SMXZF for $12 \mathrm{~h}$ before treatment with $10 \mathrm{ng} / \mathrm{mL}$ TNF-a (Sigma-Aldrich, St Louis, MO, USA) for $30 \mathrm{~min}$. The PC12 cells were treated with $10 \mu \mathrm{g} / \mathrm{mL}$ SMXZF for $12 \mathrm{~h}$ before treatment with $100 \mu \mathrm{mol} / \mathrm{L} \mathrm{H}_{2} \mathrm{O}_{2}$ (Sigma-Aldrich, St Louis, 
$\mathrm{MO}, \mathrm{USA}$ ) for $12 \mathrm{~h}$. The control groups were treated without SMXZF/TNF-a or SMXZF/ $\mathrm{H}_{2} \mathrm{O}_{2}$.

\section{Western blotting analysis}

EA.hy926 ECs and PC12 cells were lysed with $40 \mu \mathrm{L}$ of RIPA ice-cold lysis buffer (Vazyme Biotech, Nanjing, China) supplemented with protease inhibitor (Vazyme Biotech, Nanjing, China). As reported ${ }^{[29]}$, equal amounts of proteins were separated on a $12.5 \%$ SDS-PAGE and were transferred to PVDF membrane (Millipore Corporation, Billerica, MA, USA). The membranes were blocked with 3\% BSA in TBS/T and were stained with primary antibodies against p50, p65, IKKa, IKK $\beta$, p-p50, p-p65, p-IKKa, and p-IKK $\beta$ (dilution 1: 1000, 1:1000, $1: 1000,1: 1000,1: 800,1: 600,1: 800$, and 1:800, respectively; Cell Signaling Technology, Boston, MA, USA) and Nrf2, HO-1, and GAPDH (dilution 1:1000, 1:1000, and 1:4000, respectively; Bioworld Technology, Louis Park, MN, USA) overnight at $4{ }^{\circ} \mathrm{C}$. The membranes were then probed with peroxidase conjugated secondary antibody at a 1:8000 dilution (Bioworld Technology, Louis Park, MN, USA). The antigen-antibody complexes were then detected with enhanced chemiluminescence (ECL) reagent (Vazyme Biotech, Nanjing, China). The immunoreactive bands were visualized by the ChemiDoc ${ }^{\mathrm{TM}} \mathrm{MP}$ System (Bio-Rad, Hercules, CA, USA) and analyzed using Image $\mathrm{Lab}^{\mathrm{TM}}$ Software (version 4.1, Bio-Rad).

\section{Statistical analysis}

All experiments were performed in triplicate and the data are expressed as the mean \pm standard deviation. Statistical significance was determined using one-way ANOVA, followed by Student's two-tailed $t$-test for comparisons between two groups and Dunnett's test when the data involved three or more groups. $P<0.05$ was defined as significant.

\section{Results}

\section{Drug-target interaction network}

The general network properties of the drug-target network are shown in Figure 2A. Apparently, the drug-target network was dense and the combination targeted a large number of proteins. The degree and betweenness were the node topological and central indexes, corresponding to the evaluation of the regulatory relevance of the nodes. The most elementary characteristic of a node was its degree, which told us how many links the node had to other nodes. Betweenness relies on the identification of the shortest paths and measures the number of them that pass through a node. Generally, nodes with higher degree and betweenness were likely to be strongly connected in networks from a range of different domains ${ }^{[30]}$. In this network, nodes were scored with very high degree and betweenness (2.871 and 1584.43, respectively), suggesting a central regulatory role. Then, based on the high-degree nodes, we excluded nine proteins with little biological meaning in the functional annotation chart by Biocarta analysis.

\section{Target-pathway interaction network}

Three main functional annotation clusters ranked by the Biocarta functional annotation cluster tool are presented in Figure 2B. The functional annotation cluster classified the groups of similar annotations according to their enrichment scores. The enrichment scores for three main clusters of genes were 2.96, 2.08 and 1.63 (Table 1). The relevant proteins in the highest scored cluster were associated with the activation of the transcription nucleus factor signaling pathway, including NF-kB activation by nontypeable Hemophilus influenzae, the

Table 1. The clustered pathways in the network.

Annotation Cluster 1

Enrichment Score: 2.96

Annotation Cluster 2

Enrichment Score: 2.082
h_nthiPathway: NF-kB activation by Nontypeable Hemophilus Influenzae

h_RacCycDPathway: Influence of Ras and Rho Proteins on $\mathrm{G}_{1}$ to $S$ Transition

h_nfkbPathway: NF-kB Signaling Pathway

h_RELAPathway: Acetylation and Deacetylation of RelA in the Nucleus

h_keratinocytePathway: Keratinocyte Differentiation

h_41bbPathway: The 4-1BB-dependent Immune Response

h_il1rPathway: Signal Transduction through IL-1R

h_RacCycDPathway: Influence of Ras and Rho Proteins on $\mathrm{G}_{1}$ to $S$ Transition

h_tall1Pathway: TACI and BCMA Stimulation of B Cell Immune Responses

h_arenrf2Pathway: Oxidative Stress Induced Gene Expression via Nrf2

h_atmPathway: ATM Signaling Pathway

h_ceramidePathway: Ceramide Signaling Pathway

h_tollPathway: Toll-Like Receptor Pathway

h_nfatPathway: NFAT and Hypertrophy of the Heart (Transcription in the broken heart)
4.88

3.71

1.83

1.42

4.70

4.49

4.37

3.71

3.60

3.05

2.96

2.88

2.80

2.80

1.94

1.63

1.30
Annotation Cluster 3

Enrichment Score: 1.63
h_cytokinePathway: Cytokine Network

h_inflamPathway: Cytokines and Inflammatory Response

h_stemPathway: Regulation of Hematopoiesis by Cytokines

8

81

2

70

7

7


A

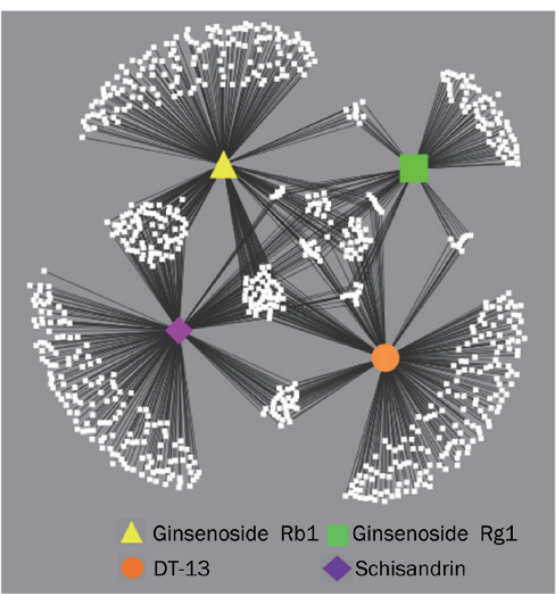

B

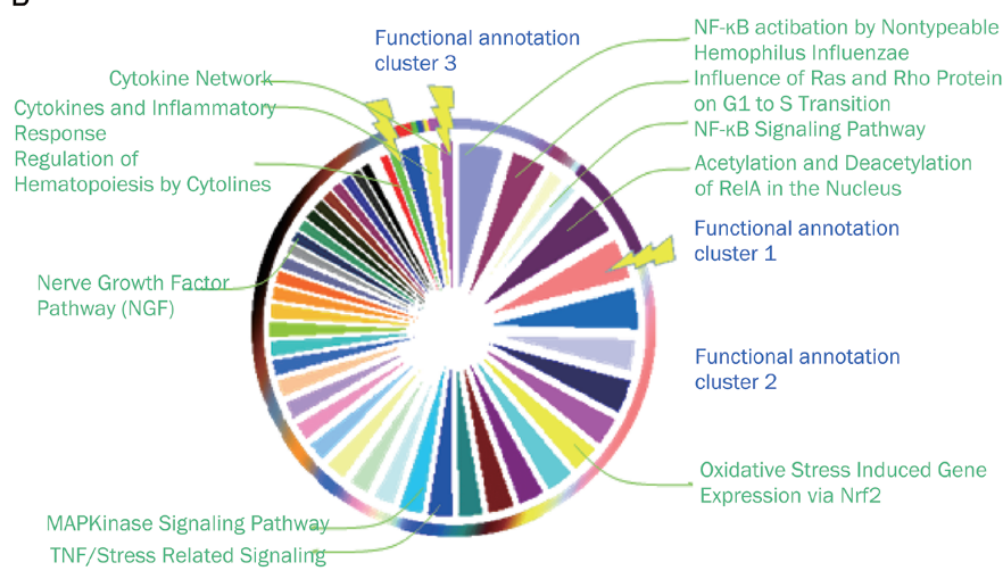

C

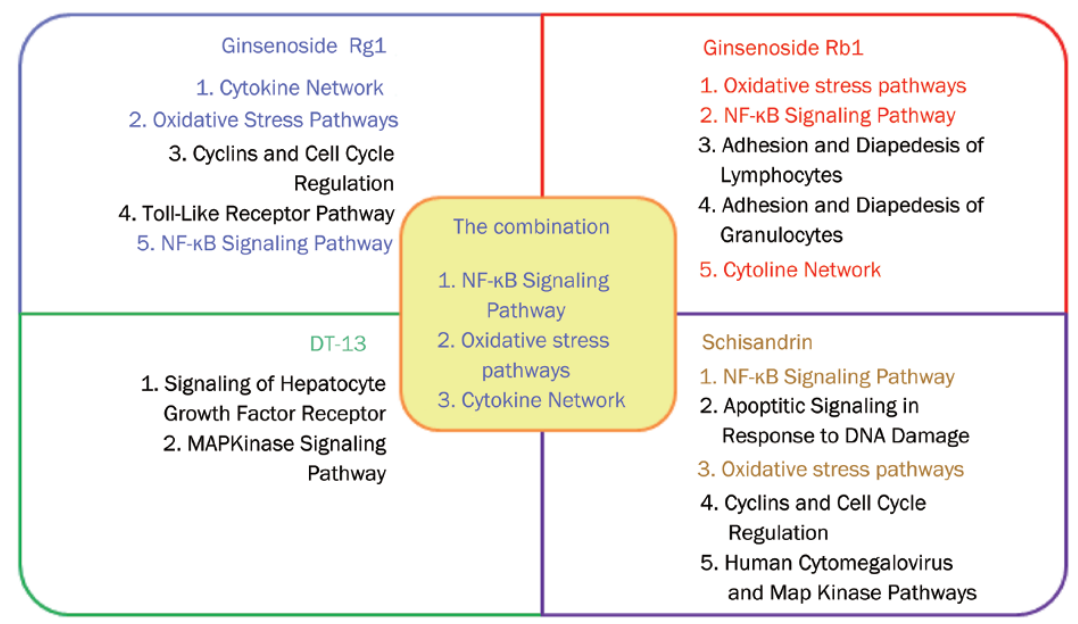

Figure 2. The drug-target network and Biocarta functional annotation analysis. (A) The drug-target network. The white parallelogram represents proteins related to cardio-cerebral ischemia in the combined TCM. The yellow triangle represents ginsenoside Rb1; the orange circle represents DT13; the purple diamond represents schisandrin; the green square represents ginsenoside Rg1. (B) Three main functional annotation clusters and main signaling pathways. The proportional areas of the pie chart are according to -lg ( $P$-value). $(\mathrm{C})$ Three main functional annotation clusters and the respective main signaling pathways for the compounds.

influence of Ras and Rho proteins on the $\mathrm{G}_{1}$ to $\mathrm{S}$ transition, the NF-kB signaling pathway and the acetylation and deacetylation of RelA in the nucleus pathways. Signaling pathways related to oxidative stress, MAPK, and nerve growth factor made up the second cluster of main pathways capable of modulating neuroprotective, anti-stress and anti-oxidative mechanisms. The third cluster had the lowest enrichment cluster score, comprising pathways related to cytokines.

\section{The relationship between the compounds and the target-pathway} interaction network

Based on the Biocarta functional annotation clusters, the four compounds from SMXZF have distinct functions in corresponding pathways (Figure 2C). Ginsenoside Rg1 affected a sequence of pathways including the cytokine network, oxidative stress pathways, cyclins and cell cycle regulation and the NF-кB signaling pathway. Ginsenoside Rb1 influenced the following pathways: the oxidative stress pathways, the NF-kB signaling pathway, the adhesion and diapedesis of lymphocytes, and the cytokine network pathway. DT-13 only influenced two clustered pathways, including the signaling of the hepatocyte growth factor receptor and the MAPKinase signaling pathway. Schisandrin interacted with the NF-kB signaling pathway, apoptotic signaling in response to DNA damage and the oxidative stress pathway.

\section{Evaluation of the important proteins}

The pathways contained in the highest scored clusters may be the interaction pathways most likely to exert their combined effects against cardio-cerebral ischemic diseases. Therefore, we focused on the highest scoring clusters that were associated with the activation of the nuclear transcription factor signaling pathway. Analysis based on functional annotation clusters represented important proteins in the signaling pathways. We found 24 proteins in the functional annotation clusters that were highly associated with each other (Table 2). For example, 
Table 2. The important proteins contained in the drug-target network.

Important proteins

Protein kinase $\mathrm{C}$ inhibitor 2

Nuclear factor, erythroid derived 2, like 2

Tumor necrosis factor (ligand) superfamily, member 13

Mitogen-activated protein kinase 11

Phospholipase A2, membrane associated

Toll-like receptor 4

Interleukin 1, beta

Interleukin 8

Interleukin 10

BCL2-associated $X$ protein

FAS (TNF receptor superfamily member 6 )

Actin, alpha 1, skeletal muscle

Tumor protein $\mathrm{p} 53$

NF-kB p65delta3

Interleukin 6 (interferon, beta 2)

Interleukin 4

Cyclin-dependent kinase inhibitor 1A (p21, Cip1)

IKK-alpha

Nuclear factor of $\mathrm{k}$ light polypeptide gene enhancer in B-cells 1

Mitogen-activated protein kinase 1

Jun (proto-oncogene)

Mitogen-activated protein kinase 8

Mitogen-activated protein kinase 14

Mitogen-activated protein kinase 3
Term frequence
40

39

35

30

25

24

16

8

7

7

7

7

6

5
IKK alpha, NF-kB p65 delta3, toll-like receptor 4, and the nuclear factor of the kappa light polypeptide gene enhancer in B cells were highly associated in the functional annotation clusters. Figure $3 \mathrm{~A}-\mathrm{C}$ depicts the graphical representations of the relative positions of the terms in the gene ontology hierarchy, with the degree of significance indicated according to color (white nodes are not significant; orange nodes are significant). The major cellular component ontologies (Figure 3A) were nuclear parts, the interleukin 6 receptor complex, the I-kappaB/NF-kappaB complex, the death-inducing signaling complex and the I-kappaB kinase complex. This was further supported by the molecular function ontologies that were significantly enriched in terms such as interleukin 1, 4, 6, and 10 receptor binding and cytokine receptor binding (Figure 3B). Additionally, the enriched biological process ontologies were dominated by a variety of NF-kB cascades, as well as the regulation or negative regulation of NF-kB imported into the nucleus and other regulations of the NF- $\mathrm{KB}$ cascade (Figure 3C).

\section{The association with related diseases}

Figure 4 presents a summary of related disease classes for the important proteins in the genetic association database. The database is an archive of human genetic association studies of complex diseases and disorders. This indicated that the important proteins were closely correlated with those associated with other diseases such as vision, renal and metabolic diseases, although they exerted functions on the cardio-cere- bral ischemic diseases.

\section{Experimental validation}

As illustrated in Figure 5, to verify the results from the target-pathway interaction network, we assessed whether SMXZF modulated the NF-кB signaling pathway. The Western blotting results showed that both phospho-NF-kB/p50 and phospho-NF-kB/p65 were increased by treatment with $10 \mathrm{ng} / \mathrm{mL}$ TNF-a for $30 \mathrm{~min}$, which could be attenuated by pretreatment with $0.1-10 \mu \mathrm{g} / \mathrm{mL}$ of SMXZF (Figure 5A and B). Moreover, we also found that the up-regulation of phosphoIKKa and phospho-IKK $\beta$ were induced by TNF- $\alpha$, while 0.1$10 \mu \mathrm{g} / \mathrm{mL}$ SMXZF could down-regulate phospho-IKKa/ $\beta$ (Figure 5C and D).

In addition, we further investigated whether SMXZF could regulate the Nrf2/HO-1 pathway in PC12 cells. Protein levels of Nrf2 in the nucleus and its downstream protein HO-1 were measured. As shown in Figure 6A, a low level of Nrf2 was detected in PC12 cells treated with $100 \mu \mathrm{mol} / \mathrm{L} \mathrm{H}_{2} \mathrm{O}_{2}$ for $12 \mathrm{~h}$, while cells pretreated with SMXZF $(10 \mu \mathrm{g} / \mathrm{mL})$ resulted in a significant increase in Nrf2 nuclear protein levels. Meanwhile, compared to the cells exposed to $100 \mu \mathrm{mol} / \mathrm{L} \mathrm{H}_{2} \mathrm{O}_{2}$ only, pretreatment with SMXZF significantly increased the expression of HO-1 (Figure 6B). These results validated the regulation of the Nrf2/HO-1 pathway by SMXZF in the exertion of antioxidant ability, which had the same effects as the positive drug NAC.

\section{Discussion}

It is well known that the complexity of ingredients, unknown targets and underlying mechanisms restrict the development of TCM. Recently, network pharmacology has offered a new method for studying the molecular mechanisms of TCM. However, many existing methodologies require significant computational knowledge and complicated algorithms for data analysis and manipulation. To increase the utility and availability of this method for beginners, it is necessary to develop some simplified network-based applications that are equally useable for cross-disciplinary users. Therefore, we proposed a target-pathway interaction network (a drug-target network combined with a pathway interaction network) to discern the targets and potential pathways of active components from a TCM formula. Then, we applied this relatively simple approach to the combination of four effective components derived from ShengMai preparation to explore its efficacy mechanism in treating cardio-cerebral ischemic disease.

We grouped the functional annotation clusters into three groups according to their enrichment scores using the Biocarta functional annotation tool, which uses a novel algorithm to measure relationships among the annotation terms based on the degrees of their co-association genes and took advantage of the well-known Biocarta pathways and displayed genes to facilitate biological interpretations in the network context. The proteins in each cluster are highly associated with each other. The most important proteins termed in the highest scored cluster were associated with NF-kB activation and the NF- $\mathrm{kB}$ 
A

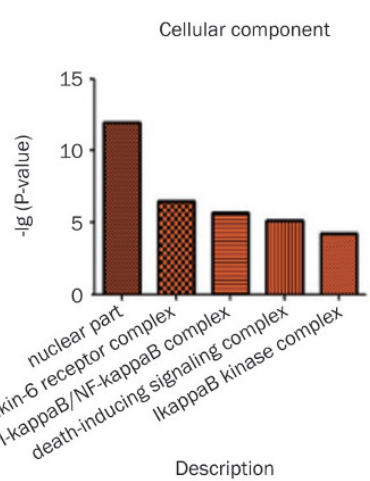

B

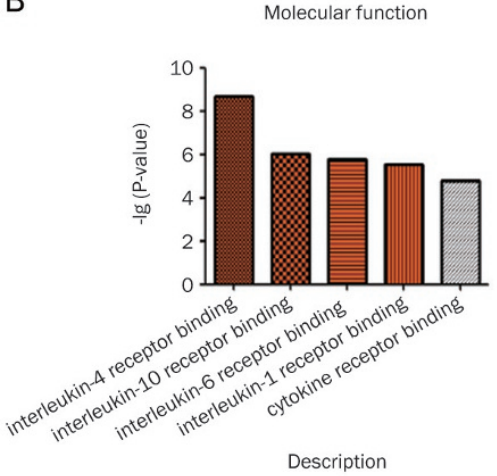

Description

C

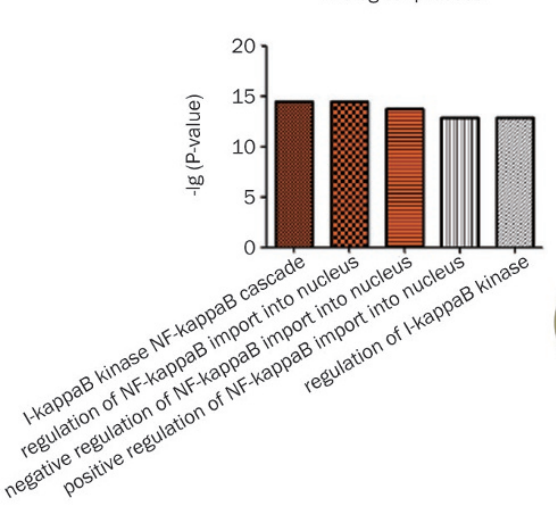

Description
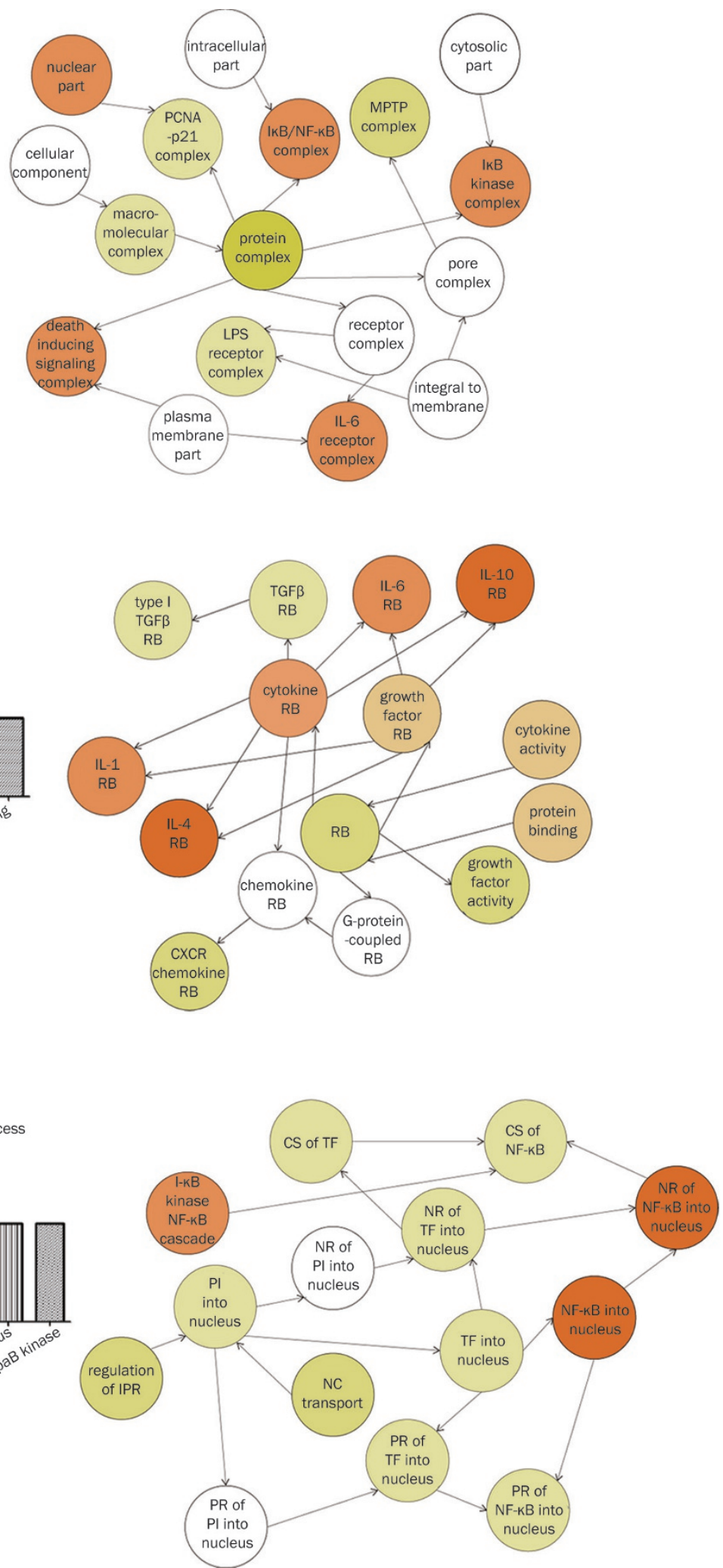

Figure 3. Statistical analysis of gene ontology metadata. Statistical analysis of the gene ontology classification was performed using the BiNGO plugin in cytoscape. The left picture is a graphical representation of top 5 scored -lg ( $P$-value) significantly enriched ontologies, presented as the cellular component (A), molecular function (B) and biological process (C). The right picture is the enlarged figures including different numbers of top ontologies in the analysis. The orange-marked ontologies in the left are contained in the corresponding right figures. IPR: intracellular protein transport, PR: positive regulation, NR: negative regulation, PI: protein import, NC: nucleocytoplasmic, TF: transcription factor, CS: cytoplasmic sequestering, TGF: transforming growth factor, RB: receptor binding. 


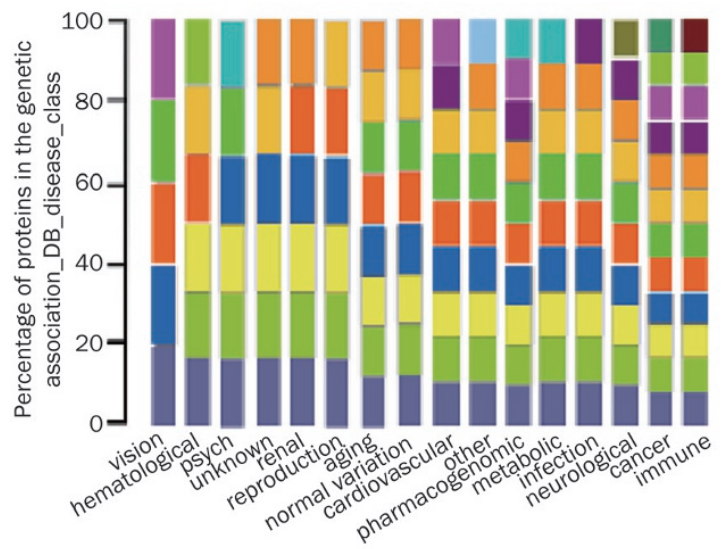

nuclear factor (erythroid-derived 2)-like 2

actin, alpha 1, skeletal muscle

tumor necrosis factor (ligand)

superfamily, member13

v v-rel reticuloendotheliosis viral

oncogene homolog A (avian)

- phospholipase A2, group IVA

(cytosolic, calcium-dependent)

BCL-2-associated X protein

- cyclin-dependent kinase inhibitor 1A (p21, Cip1)

nuclear factor of kappa light polypeptide gene enhancer in B-cells 1

- interleukin8

- Fas (TNF receptor superfamily, member 6)

II tumor protein $\mathrm{p} 53$

- toll-like receptor 4

- interleukin 6 (interferon, beta 2)

팔 interleukin 10

interleukin 4

interleukin 1, beta

Figure 4. The association of the important proteins with genetic database disease classes.

A
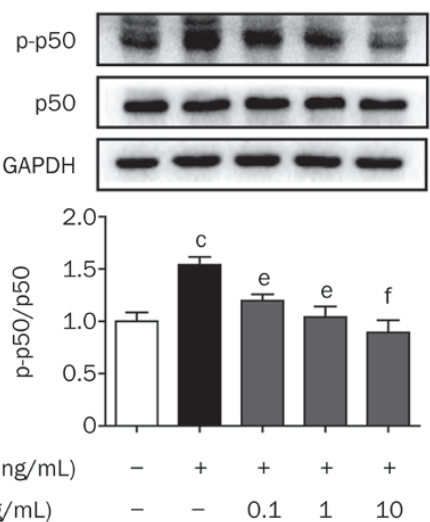

C
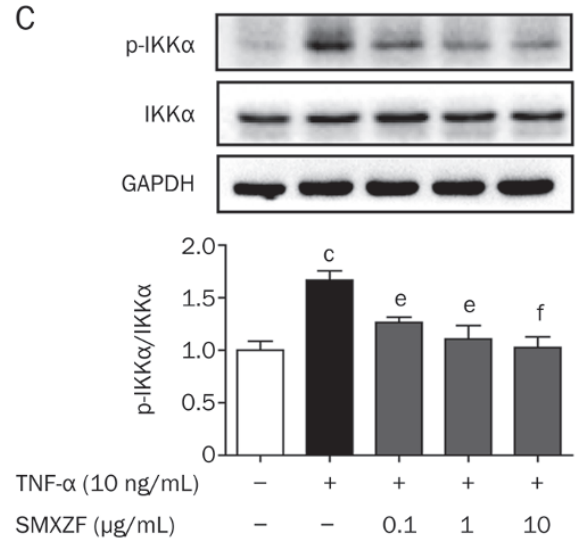

B
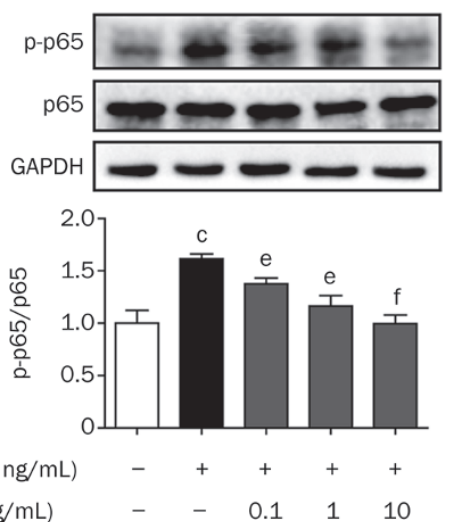

D
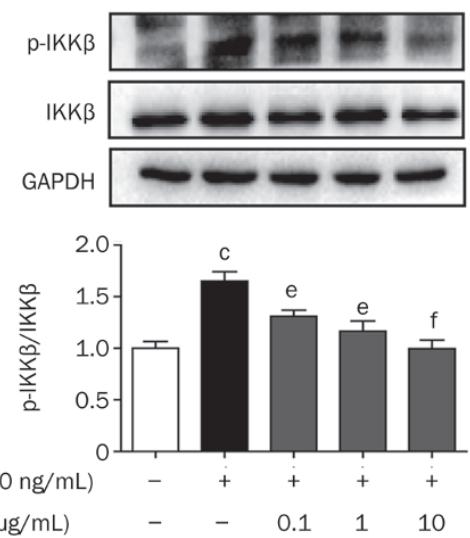

Figure 5. The effects of SMXZF on the NF-KB signaling pathway induced by TNF- $\alpha$ in EA.hy926 cells. EA.hy926 cells were pretreated with SMXZF (0.1-10 $\mu \mathrm{g} / \mathrm{mL}$ ) for $12 \mathrm{~h}$ and then exposed to TNF- $\alpha$ for $30 \mathrm{~min}$. Protein expressions were tested by Western blot. (A) Protein expressions of p50 and phospho-p50 using GAPDH as the loading control. (B) Protein expressions of p65 and phospho-p65 using GAPDH as the loading control. (C) Protein expressions of IKK $\alpha$ and phospho-IKK $\alpha$ using GAPDH as the loading control. (D) Protein expressions of IKK $\beta$ and phospho-IKK $\beta$ using GAPDH as the loading control. The results were obtained from three independent experiments and were expressed as the mean \pm SD. ${ }^{\circ} P<0.01$ vs Control group without TNF- $\alpha,{ }^{e} P<0.05,{ }^{\text {f }} P<0.01$ vs group treated with TNF- $\alpha$ alone. 
A
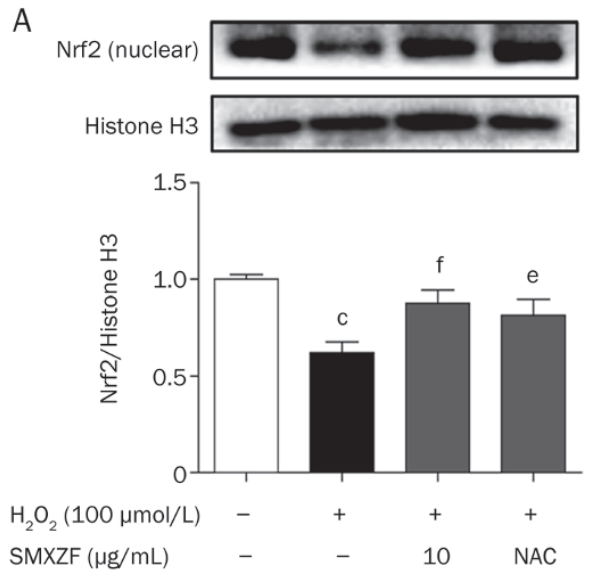

B
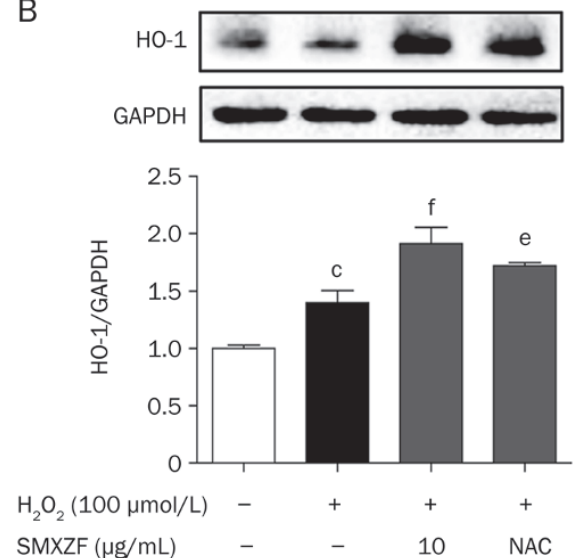

Figure 6. The effect of SMXZF on the Nrf2/HO-1 pathway induced by $\mathrm{H}_{2} \mathrm{O}_{2}$ in PC12 cells. PC12 cells were pretreated with $10 \mu \mathrm{g} / \mathrm{mL} \mathrm{SMXZF} \mathrm{and} 500$ $\mu \mathrm{mol} / \mathrm{L} \mathrm{NAC}$ for $12 \mathrm{~h}$ and were then exposed to $100 \mu \mathrm{mol} / \mathrm{L} \mathrm{H}_{2} \mathrm{O}_{2}$ for $12 \mathrm{~h}$. Protein expressions were tested by Western blot. (A) Protein expressions of Nrf2 using Histone $\mathrm{H} 3$ as the loading control. (B) Protein expressions of HO-1 using GAPDH as the loading control. The results were obtained from three independent experiments and were expressed as the mean \pm SD. ${ }^{\mathrm{C}} P<0.01$ vs Control group without $\mathrm{H}_{2} \mathrm{O}_{2},{ }^{\mathrm{e}} P<0.05,{ }^{\mathrm{f}} P<0.01$ vs group treated with $\mathrm{H}_{2} \mathrm{O}_{2}$ alone.

signaling pathway. Various pharmacological studies showed that the transcription factor NF-kB plays a key role in cell survival in the nervous system and acts as a key regulator of the inflammatory process ${ }^{[3] 1}$. Inhibiting the activity of NF-kB also protects the heart against myocardial ischemia reperfusion injury ${ }^{[32]}$. SMXZF most likely made up an interlinked network so that these building blocks of the body could have function as a whole. In this study, we observed the effect of SMXZF on the expression and activation of NF-kB p50, NF-kB p65 and IKKa, IKK $\beta$ in TNF- $\alpha$ induced EA.hy926 ECs, confirming the predictive results and providing an explanation of the molecular mechanisms of cardio-cerebral ischemic diseases. In our previous study, we found that ginsenoside Rg1 could protect against hydrogen peroxide-induced cell death in PC12 cells via inhibiting NF-kB activation ${ }^{[33]}$. We also confirmed that schisandrin inhibited the upregulation of IKKa, and also exerted significant inhibition on the expression and phosphorylation of p50, p65 and IKK $\beta$ induced by $\mathrm{H}_{2} \mathrm{O}_{2}$ in PC12 cells ${ }^{[2]}$. Meanwhile, pharmacological studies have shown that ginsenoside Rb1 exerts significant NF-KB inhibitory effects ${ }^{[34]}$.

Signaling pathways related to oxidative stress, MAPK, and nerve growth factor (NGF) are the second main pathways. Several pathways such as oxidative stress that induced gene expression via Nrf2, TNF/stress related signaling, the MAPKinase signaling pathway, angiotensin II mediated activation of the JNK pathway via Pyk2 dependent signaling, and the NGF pathway were clustered to similar functional annotations. Oxidative stress of the neuron and the neuroprotective mechanism of the combination is a contribution to research on the mechanisms of cardio-cerebral ischemia ${ }^{[35]}$. Nrf2 activation and subsequent cytoprotective gene induction promote the restoration of balance between oxidants and antioxidants after oxidative insult. It was reported that a great increase in the nuclear level of Nrf2 followed by a decrease to the basal level or even lower occurs in $\mathrm{H}_{2} \mathrm{O}_{2}$-induced $\mathrm{PC} 12$ cells ${ }^{[36]}$. Our stud- ies showed that SMXZF induced Nrf2 nuclear translocation as well as HO-1 expression, which demonstrated that SMXZF exerted antioxidant capacity via the Nrf2/HO-1 pathway. The validated functions of the combination of four effective compounds complied with the predicted action mechanism related to oxidative stress. Schisandrin was previously reported to have anti-inflammatory activity by the upregulation of HO-1 via the Nrf-2 signaling pathways ${ }^{[37]}$, ginsenoside Rb1 could protect against oxidative stress by increasing HO-1 expression through the PI3K/Akt/Nrf2-dependent pathway and ginsenoside Rg1 could also activate the Nrf2 pathway to exert antioxidative actions ${ }^{[38,39]}$. The third cluster was also the cluster with the lowest enrichment score and comprised pathways related to cytokines. There were three pathways, including the cytokine network, cytokines and the inflammatory response and regulation of hematopoiesis by cytokine pathways. Cytokines constituted the foundation for shock movements from ischemia to inflammation ${ }^{[40]}$. Ginsenoside Rg1 and Rb1 participated in the inhibitory effect on interleukin and contributed to the neuroprotective effect in cerebral ischemia ${ }^{[41,42]}$.

The combination of four effective compounds was predicted to exert incorporated functions on three main pathway clusters. The respective functions of each compound were exerted via different pathways. Ginsenoside Rg1 acted on the cytokine network and oxidative stress pathways. Ginsenoside Rb1 was predicted to have the strongest functions in the NF- $\mathrm{KB}$ signaling pathway. DT-13 mainly acted on the signaling of the hepatocyte growth factor receptor and the MAPKinase signaling pathways. Schisandrin acted on the NF-KB signaling pathway. The clustered pathways for each compound also had similarities. The three main pathways had higher frequencies than most of the pathways, indicating that they might comprise the corporative functions in their entirety. By inducing different effects on different pathways, the four compounds achieve synergistic effects on some of the main pathways 
against the disease.

A given protein list found that 24 of the high-degree nodes were involved in the highest annotation cluster pathway. MAPK are involved in directing cellular responses to a diverse array of stimuli, such as mitogens, heat shock and pro-inflammatory cytokines. Mitogen-activated protein kinases 3, 14 and 8 regulate proliferation, gene expression, mitosis, cell survival, apoptosis and many other cell functions ${ }^{[43]}$. The function of the immune system depends largely on interleukins and the majority of interleukins promote the development and differentiation of T, B and hematopoietic cells. Interleukin 6, interleukin 4 , interleukin 8 , interleukin 10 and interleukin 1 play essential roles in the final differentiation of B cells, as well as inducing myeloma growth and nerve cell differentiation $^{[44]}$. Transcription factors make up the single largest family of human proteins and perform their functions alone or with other proteins in a complex. The action mechanism of these key proteins may be that they affect the integrated biological network rather than one target. The entire biological network would be unstable in an unhealthy state when the key targets are extracted from the network. Alternatively, the unhealthy state would be returned to normal when multiple key proteins targeted in the network.

Gene ontology has been widely accepted as the ontology for designating structural controlled vocabulary terms to describe gene products in any organism. The vocabulary terms are used as attributes of genes in databases from a wide variety of species and facilitate computational queries across species. Gene ontology consists of three separate ontologies to describe the attributes of gene products or gene product groups. Molecular function describes what a gene product does at the biochemical level and biological process refers to a biological objective to which a gene product contributes, while cellular component refers to the place in the cell where a gene product is found. The three annotation levels were all consistent with signaling pathways involved in the functional annotation clusters, which were all associated with the NF- $\mathrm{kB}$ complex and the interleukin receptor contained in the pathway clusters. This also verified the validity of the signaling pathway clusters and illuminated the action mechanism from the perspective of gene ontology.

The association between target proteins and other diseases was also discussed. The pharmacological functions of TCMs are more widespread than those of western drugs. TCMs play a role not only in the treatment of diseases but also in the regulation of other closely related complicating diseases. The identified key proteins are also related to other diseases. The gene expression of interleukin 1 beta was investigated in a transgenic mouse model of the inflammatory process and its efficacy as an anti-inflammatory drug was evaluated in vivo ${ }^{[45]}$. Toll-like receptor- 4 was determined to be a cellular sentinel for acute renal damage by targeting the intracellular pathways ${ }^{[46]}$. Anti-inflammatory cytokines interleukin 4 and interleukin 10 play roles in the neurodegenerative process of CreutzfeldtJakob disease ${ }^{[47]}$.
In this study, network pharmacology was applied to investigate the combination of four bioactive ingredients derived from ShengMai preparations to allow us to understand the underlying molecular mechanisms from the active chemical, biological pathways, target proteins, and disease networks perspectives. Certainly, our approach has some limitations. First, our approach could not discriminate whether an ingredient could directly bind a target or whether it could indirectly affect the target. Secondly, our approach might suffer from annotation bias, which is also a limitation of most functional annotation-based methods ${ }^{[48,49]}$. Consequently, the strategy might function poorly for compounds lacking approved gene association annotations. Moreover, the results obtained by this method were completely dependent on the database. Therefore, in combination with experimental techniques, this method plays an important role in understanding the molecular mechanisms of TCM.

\section{Conclusion}

In this study, we proposed a simple and integrated method for predicting the possible molecular mechanism of the combination of four effective compounds derived from ShengMai preparations against cardio-cerebral ischemic diseases based on an integrated target-pathway interaction network. Using this method, we inferred links between active ingredients and cardio-cerebral ischemic diseases through molecular targets and several key signaling pathways. Based on the most predictive potential pathway, the NF-kB signaling pathway and the related oxidative stress pathway were further confirmed by experimental verification in a cell model. Further studies need to conduct more tests of the effects of bioactive ingredients on other pathways and their interactions. Our findings may also provide clues about the mechanisms of TCM formulas with multiple active components.

\section{Acknowledgements}

This research work was supported by the National Natural Science Foundation of China (№ 81274004), 2011 Program for Excellent Scientific and Technological Innovation Team of Jiangsu Higher Education, a Project Funded by the Priority Academic Program Development of Jiangsu Higher Education Institutions, the Project Program of the State Key Laboratory of Natural Medicines, China Pharmaceutical University (No JKGZ201107), and the Graduate Student Scientific Research Innovation Plan of Jiangsu Higher Education Institutions (CXLX13_30).

\section{Author contribution}

Fang LI, Yan-ni LV, Jun-ping KOU and Bo-yang YU conceived and designed the study; Fang LI, Ke-feng ZHAI, Kai SHEN and Hong-lin CHEN performed the cell experiments; Yan-ni LV and Yi-sha TAN analyzed the data and performed the network analysis; Fang LI, Yan-ni LV and Yi-sha TAN wrote the manuscript; Jun-ping KOU and Bo-yang YU revised the paper. 
All the authors read and approved the final manuscript.

\section{References}

1 Herrick TM, Million RP. Tapping the potential of fixed-dose combinations. Nat Rev Drug Discov 2007; 6: 513-4.

2 Barabasi AL, Oltvai ZN. Network biology: Understanding the cell's functional organization. Nat Rev Genet 2004; 5: 101-3.

3 Verpoorte R, Choi YH, Kim HK. Ethnopharmacology and systems biology: A perfect holistic match. J Ethnopharmacol 2005; 100: 53-6.

4 Li S, Zhang B, Zhang NB. Network target for screening synergistic drug combinations with application to traditional Chinese medicine. BMC Syst Biol 2011; 5: S10.

5 Gu JY, Zhang H, Chen LR, Xu S, Yuan G, Xu XJ. Drug-target network and polypharmacology studies of a traditional Chinese medicine for type II diabetes mellitus. Comput Biol Chem 2011; 35: 293-7.

6 Jiang $\mathrm{M}$, Chen YK, Zhang YC, Chen L, Zhang N, Huang T, et al. Identification of hepatocellular carcinoma related genes with $\mathrm{k}$-th shortest paths in a protein-protein interaction network. Mol Biosyst 2013; 9: 2720-8.

7 Wu XB, Jiang R, Zhang MQ, Li S. Network-based global inference of human disease genes. Mol Syst Biol 2008; 4: 189.

8 Zhang YC, Lu BJ, Zhao MH, Rong YZ, Chen RM. Effect of Shengmai injection on vascular endothelial and heart functions in patients with coronary heart disease complicated with diabetes mellitus. Chin J Integr Med 2008; 14: 281-5.

9 Konishi T. Brain oxidative stress as basic target of antioxidant traditional oriental medicines. Neurochem Res 2009; 34: 711-6.

10 Ichikawa H, Wang L, Konishi T. Prevention of cerebral oxidative injury by post-ischemic intravenous administration of Shengmai San. Am J Chin Med 2006; 34: 591-600.

11 Wang YH, Qiu C, Wang DW, Hu ZF, Yu BY, Zhu DN. Identification of multiple constituents in the traditional Chinese medicine formula Sheng-Mai San and rat plasma after oral administration by HPLCDAD-MS/MS. J Pharm Biomed Anal 2011; 54: 1110-27.

12 Guo ZS, Cao GS, Yang HP, Zhou HN, Li L, Cao ZY, et al. A combination of four active compounds alleviates cerebral ischemia-reperfusion injury in correlation with inhibition of autophagy and modulation of AMPK/mTOR and JNK pathways. J Neurosci Res 2014; 92: $1295-$ 306.

13 Yu BY, Kou JP, Zhang J, Zhu DN, Qi J, Yan YQ. China Patent, ZL 200710132029 (5), 2007.

$14 \mathrm{Lee} \mathrm{CH}$, Kim JH. A review on the medicinal potentials of ginseng and ginsenosides on cardiovascular diseases. J Ginseng Res 2014; 38: 161-6.

15 Cheng HY, Hsieh MT, Wu CR, Tsai FH, Lu TC, Hsieh CC, et al. Schizandrin protects primary cultures of rat cortical cells from glutamate-induced excitotoxicity. J Pharmacol Sci 2008; 107: 21-31.

16 Tao J, Wang HY, Zhou H, Li SN. The saponin monomer of dwarf lilyturf tuber, DT-13, reduces L-type calcium currents during hypoxia in adult rat ventricular myocytes. Life Sci 2005; 77: 3021-30.

17 Heitzer T, Schlinzig T, Krohn K, Meinertz T, Munzel T. Endothelial dysfunction, oxidative stress, and risk of cardiovascular events in patients with coronary artery disease. Circulation 2001; 104: 26738.

18 Jin HM, Liu QH, Cao X, Wu ZH, Zhang GP, Zhang M, et al. Dysfunction of microvascular endothelial cells induced by tumor necrosis factor (TNF $\alpha$ ): cellular and molecular mechanism. Clin Hemorheol Micro 2000; 23: 109-12.

19 Green SH. The use of PC12 cells for the study of the mechanism of action of neurotrophic factors: Signal transduction and programmed cell death. Methods 1995; 7: 222-37.

20 Yanovsky I, Finkin-Groner E, Zaikin A, Lerman L, Shalom H, Zeeli S, et al. Carbamate derivatives of indolines as cholinesterase inhibitors and antioxidants for the treatment of Alzheimer's disease. J Med Chem 2012; 55: 10700-15.

21 ArunaDevi R, Lata S, Bhadoria BK, Ramteke VD, Kumar S, Sankar P, et al. Neuroprotective effect of 5, 7, 3', 4', 5'-pentahydroxy dihdroflavanol-3-0-(2"-0-galloyl)- $\beta$-d-glucopyranoside, a polyphenolic compound in focal cerebral ischemia in rat. Eur J Pharmacol 2010; 626: 205-12.

22 Liu XF, Ouyang SS, Yu B, Liu YB, Huang K, Gong JY, et al. PharmMapper server: a web server for potential drug target identification using pharmacophore mapping approach. Nucleic Acids Res 2010; 38: W609-14.

23 Cline MS, Smoot M, Cerami E, Kuchinsky A, Landys N, Workman C, et al. Integration of biological networks and gene expression data using Cytoscape. Nat Protoc 2007; 2: 2366-82.

24 Tan YS, Li F, Lv YN, Zhai KF, Chai CZ, Kou JP, et al. Study on the multitargets mechanism of YiQiFuMai powder injection on cardio-cerebral ischemic diseases based on network pharmacology. J Proteomics Computational Biol 2014; 1: 9.

25 Huang DW, Sherman BT, Lempicki RA. Bioinformatics enrichment tools: paths toward the comprehensive functional analysis of large gene lists. Nucleic Acids Res 2009; 37: 1-13.

26 Opsahl T, Agneessens F, Skvoretz J. Node centrality in weighted networks: Generalizing degree and shortest paths. Social Networks 2010; 32: 245-51.

27 Barabasi AL, Oltvai ZN. Network biology: understanding the cell's functional organization. Nat Rev Genet 2004; 5: 101-13.

28 Apic G, Ignjatovic T, Boyer S, Russell RB. Illuminating drug discovery with biological pathways. FEBS Lett 2005; 579: 1872-7.

29 Lv YN, Li SX, Zhai KF, Kou JP, Yu BY. Network pharmacology-based prediction and verification of the molecular targets and pathways for schisandrin against cerebrovascular disease. Chin J Nat Med 2014; 12: 251-8.

30 Jeong $\mathrm{H}$, Mason SP, Barabási AL, Oltvai ZN. Lethality and centrality in protein networks. Nature 2001; 411: 41-2.

31 Mattson MP. NF-kappaB in the survival and plasticity of neurons. Neurochem Res 2005; 30: 883-93.

32 Liang XM, Huang JC, Lin X, Qin FZ, Wen QW, Chen CX, et al. The effect of 17-Methoxyl-7-Hydroxy-Benzene-Furanchalcone on NF-kB and the inflammatory response during myocardial ischemia reperfusion injury in rats. J Cardiovasc Pharm 2014; 63: 68-75.

33 Liu Q, Kou JP, Yu BY. Ginsenoside Rg1 protects against hydrogen peroxide-induced cell death in PC12 cells via inhibiting NF-KB activation. Neurochem Int 2011; 58: 119-25.

34 Xing L, Jiang M, Dong LY, Gao J, Hou YY, Bai G, et al. Cardioprotective effects of the YiQiFuMai injection and isolated compounds on attenuating chronic heart failure via NF-KB inactivation and cytokine suppression. J Ethnopharmacol 2013; 148: 239-45.

35 Wang X, Cui L, Joseph J, Jiang BB, Pimental D, Handy DE, et al. Homocysteine induces cardiomyocyte dysfunction and apoptosis through p38 MAPK-mediated increase in oxidant stress. J Mol Cell Cardiol 2012; 52: 753-60.

36 Tusi SK, Ansari N, Amini M, Amirabad AD, Shafiee A, Khodagholi F. Attenuation of NF-KB and activation of Nrf2 signaling by 1,2 , 4-triazine derivatives, protects neuron-like PC12 cells against apoptosis. Apoptosis 2010; 15: 738-51.

37 Park SY, Park DJ, Kim YH, Kim YH, Kim SG, Shon KJ, et al. Upregulation of heme oxygenase-1 via PI3K/Akt and Nrf-2 signaling pathways mediates the anti-inflammatory activity of Schisandrin in 
Porphyromonas gingivalis LPS-stimulated macrophages. Immunol Lett 2011; 139: 93-101.

38 Hwang YP, Jeong HG. Ginsenoside Rb1 protects against 6-hydroxydopamine-induced oxidative stress by increasing heme oxygenase-1 expression through an estrogen receptor-related PI3K/ Akt/Nrf2-dependent pathway in human dopaminergic cells. Toxicol Appl Pharm 2010; 242: 18-28.

39 Li JP, Gao Y, Chu SF, Zhang Z, Xia CY, Mou Z, et al. Nrf2 pathway activation contributes to anti-fibrosis effects of ginsenoside Rg1 in a rat model of alcohol-and $\mathrm{CCl}_{4}$-induced hepatic fibrosis. Acta Pharmacol Sin 2014; 35: 1031-44.

40 Girard S, Sebire H, Brochu ME, Briota S, Sarret P, Sebire G. Postnatal administration of IL-1Ra exerts neuroprotective effects following perinatal inflammation and/or hypoxic-ischemic injuries. Brain Behav Immun 2012; 26: 1331-9.

41 Huang J, Ding L, Shi D, Hu JH, Zhu QG, Gao S, et al. Transient receptor potential vanilloid-1 participates in the inhibitory effect of ginsenoside Rg1 on capsaicin-induced interleukin-8 and prostaglandin $E_{2}$ production in HaCaT cells. J Pharm Pharmacol 2012; 64: 252-8.

42 Zhu J, Jiang Y, Wu L, Lu T, Xu G, Liu X. Suppression of local inflammation contributes to the neuroprotective effect of ginsenoside $\mathrm{Rb} 1$ in rats with cerebral ischemia. Neuroscience 2012; 202: 342-51.

43 Pearson G, Robinson F, Gibson TB, Xu BE, Karandikar M, Berman K, et al. Mitogen-activated protein (MAP) kinase pathways: regulation and physiological functions 1. Endocr Rev 2001; 22: 153-83.

44 Brocker C, Thompson D, Matsumoto A, Nebert DW, Vasiliou V. Evolutionary divergence and functions of the human interleukin (IL) gene family. Human Genomics 2010; 5: 30.

45 Li LM, Fei ZL, Ren JK, Sun RL, Liu ZH, Sheng ZJ, et al. Functional imaging of interleukin 1 beta expression in inflammatory process using bioluminescence imaging in transgenic mice. BMC Immunol 2008; 9: 49.

46 Pulskens WP, Teske GJ, Butter LM, Roelofs JJ, van der Poll T, Florquin $\mathrm{S}$, et al. Toll-like receptor-4 coordinates the innate immune response of the kidney to renal ischemia/reperfusion injury. Plos One 2008; 3 : e3596.

47 Stoeck K, Bodemer M, Ciesielczyk B, Meissner B, Bartl M, Heinemann $U$, et al. Interleukin 4 and interleukin 10 levels are elevated in the cerebrospinal fluid of patients with Creutzfeldt-Jakob disease. Arch Neurol 2005; 62: 1591-4.

48 Adie EA, Adams RR, Evans KL, Porteous DJ, Pickard BS. SUSPECTS: enabling fast and effective prioritization of positional candidates. Bioinformatics 2006; 22: 773-4.

49 Kohler S, Bauer S, Horn D, Robinson PN. Walking the interactome for prioritization of candidate disease genes. Am J Hum Genet 2008; 82: 949-58. 